\title{
Small Signal Internal Voltage Transfer Measurements and White-Box Transient Calculations for Non- Standard Test Conditions of a Shell-Form Power Transformer
}

\author{
Bjørn Gustavsen ${ }^{1}$, Ariana Martins², Carlos A. Sá2 ${ }^{2}$ Luis Braña ${ }^{3}$, Ricardo Castro Lopes ${ }^{3}$, Pedro Lima ${ }^{3}$, Andrea Soto ${ }^{3}$, and \\ Mário Soares ${ }^{4}$ \\ ${ }^{1}$ SINTEF Energy Research, Postboks 4761 Sluppen, NO-7465 Trondheim, Norway \\ ${ }^{2}$ Universidade do Porto, Faculdade de Engenharia, Porto, Portugal \\ ${ }^{3}$ Efacec Energia - Máquinas e Equipamentos Eléctricos, S.A., Matosinhos, Portugal \\ ${ }^{4}$ REN - Rede Eléctrica Nacional, S.A., Lisboa, Portugal \\ E-mail: bjorn.gustavsen@sintef.no
}

\begin{abstract}
Research tests were performed on a shell-form autotransformer with a more rigorous impulse test methodology than the traditional recurrent surge oscillograph method. These tests included non-standard terminal connections with open terminals, differing from those tests defined by the international standards related with impulse testing of power transformers. The test voltage responses were obtained using voltage transfer frequency sweep measurements that were converted into time domain waveforms. The measurement results were compared against simulations by a white-box model, demonstrating satisfactory accuracy of the transient calculation tool. In addition, the sensitivity of the measurements to measuring probes length was experimentally evaluated. Reducing the lengths with $1.5 \mathrm{~m}$ only affected frequency components around $1 \mathrm{MHz}$.
\end{abstract}

Index Terms—-shell-form transformer, transient response measurements, simulations, white-box model.

\section{INTRODUCTION}

The dielectric withstand capability of the transformer windings against transient overvoltages is verified by the lightning impulse factory test [1]. In-service voltages impinging the transformer terminals are however very different from the standard impulse test voltage. The verification against internal stresses due to other voltage wave shapes and terminal conditions can only be calculated using the white-box transformer model available to the transformer designer. It has been found in CIGRE JWG A2/C4.39 [2] and A2/C4.52 (ongoing) that although the white-box models used by manufacturers are well suited for the standard lightning impulse response prediction, their accuracy still needs to be evaluated for the application of non-standard waveshapes and terminal connections.

In order to assess the white-box accuracy limitations, we performed research tests with direct measurements of frequency domain voltage transfer functions, from external terminals to critical internal points in the regulating winding. The time domain voltage response to any time domain excitation could then be determined via rational approximation and convolutions and be compared against simulations by a white-box model. While a similar approach has already been applied to core-form transformers [3], we apply in this work the approach to a shell-form transformer. We also investigate the effect of measurement lead lengths on the measured results.

\section{SHELL-FORM TRANSFORMER}

A. Transformer main data

The tested transformer is a seven leg, $800 \mathrm{MVA}$, shell-form, three-phase autotransformer. The nominal phase-to-phase voltages are $500 \mathrm{kV}, 161 \mathrm{kV}$ and $34.5 \mathrm{kV}$, for the high voltage, low voltage and tertiary voltage respectively. Each phase of the series winding was designed with 6 tap leads, for connection to a de-energized tap-changer and were available for testing. At the time of testing, the connections between phases were not done yet (neutral and delta), so all the leads were available as 3 single phase units.

\section{B. Transformer winding layout}

Each phase is built with 56 pancake coils ( 4 for tertiary winding, 24 for common winding, 28 for series winding), and 6 static shields as per Fig. 1. Minimum tap position was chosen for these tests, because it leads to a higher floating portion of tap winding, so higher voltages should be expected at tap leads P5 and P6. 


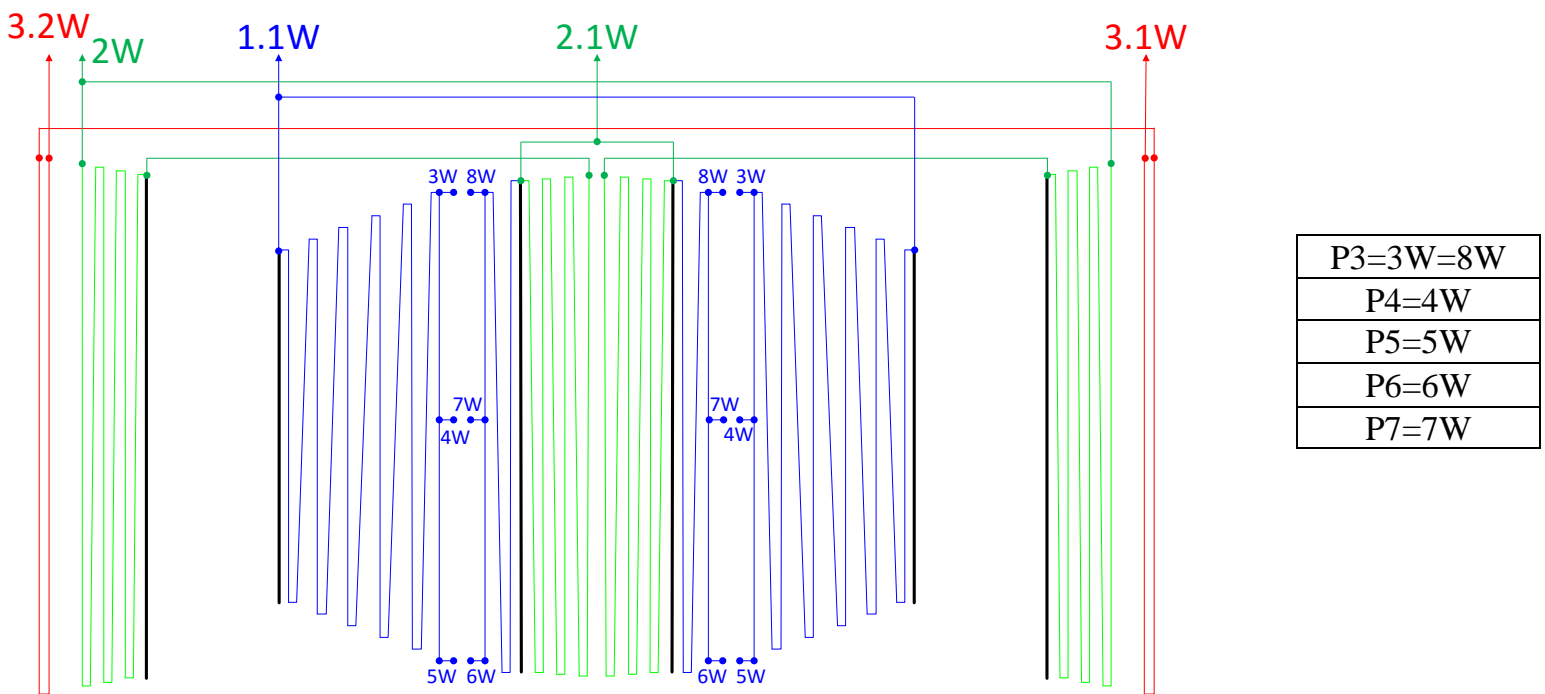

Figure 1: Left: Window cutaway for phase W (red: tertiary winding and leads, green: common winding and leads, blue: series winding and leads, black: static shields). Right: Table with the definition of the de-energized tap changer connections referring to the minimum tap position.

C. Specifics of shell-form vs core-form effect on voltage distribution

Shell-form transformer coils have a very large surface facing each other and a very small surface facing the ground (magnetic circuit, magnetic shielding and tank walls), which results in a very high series capacitance ( $\left.\mathrm{C}_{s}\right)$ and a very low capacitance to ground $\left(\mathrm{C}_{\mathrm{G}}\right)$. The higher the ratio between $\mathrm{C}_{\mathrm{S}}$ and $\mathrm{C}_{\mathrm{G}}$ the more linear will be the impulse voltage (or of any other high frequency voltage) distribution along the windings. If these voltage distributions were not close to linear, then some localized overvoltages would appear, which means that the insulating structure would have to be reinforced at some spots, and probably overdimensioned at others. As an illustration for this, one can consider the capacitances circuit in Fig. 2 (that is somewhat representative of a winding for high frequencies), where we have five elemental series capacitances and four elemental capacitances to ground. If we consider $k$ as the ratio between $C_{S}$ and $C_{G}$ and apply a high frequency sinusoidal voltage at terminal $V_{1}$, we can assess the voltage distribution at $V_{2}, V_{3}, V_{4}$ and $V_{5}$ as $k$ changes. As we can see in Fig. 3, the higher the $k$, the more linear will be the voltage distribution. For instance, if we take $C_{S}$ equal to $C_{G}(k=1)$, the voltage difference between $V_{1}$ and $V_{2}$ will be 0.618 p.u., while if $C_{S}$ is one hundred times higher than $C_{G}$ ( $k=100$ ) the same voltage difference will be 0.212 p.u. This means that for $k=1$, the voltage difference will be 2.9 times higher than for $k=100$, and thus will result in a higher dielectric stress at the respective series capacitor Cs.

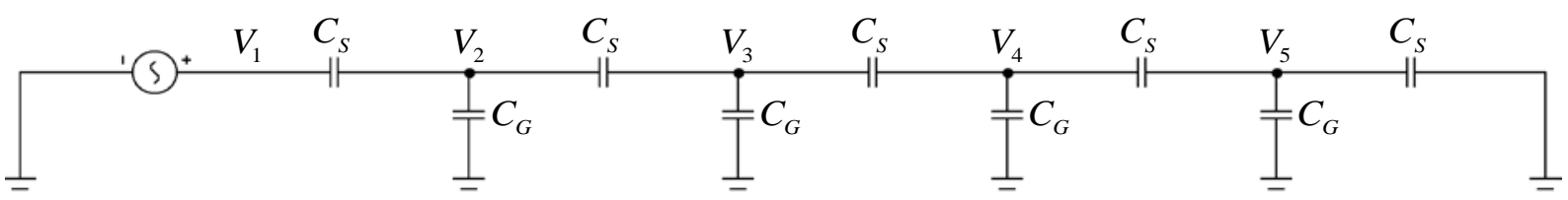

Figure 2: Example capacitances circuit ( $\mathrm{C}_{\mathrm{S}}$ : elemental series capacitance, $\mathrm{C}_{\mathrm{G}}$ : elemental capacitance to ground).

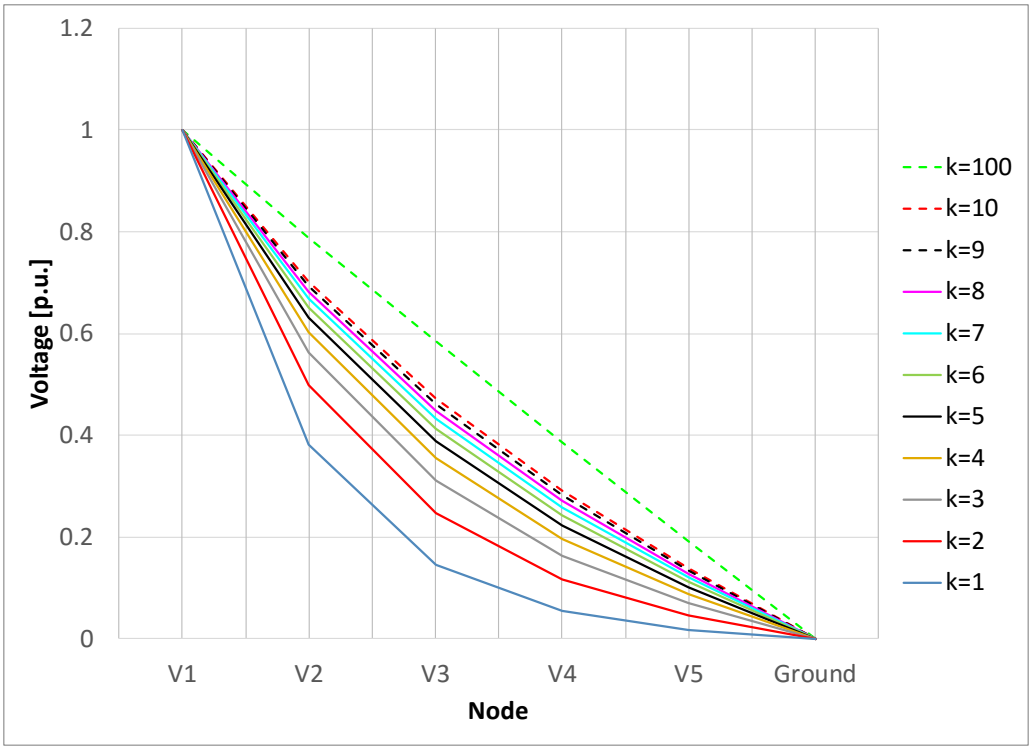

Figure 3: Voltage distribution for different $k$ factors (ratio between $\mathrm{C}_{\mathrm{S}}$ and $\mathrm{C}_{\mathrm{G}}$ ). 


\section{VOLTAGE TRANSFER MEASUREMENTS}

\section{A. Procedure}

The basic approach is to measure the voltage transfer function from one transformer terminal to a second terminal, as function of discrete frequency. The voltage transfer function $h(\omega)$ is defined as the response voltage $V_{2}(\omega)$ divided by the excitation voltage $V_{1}(\omega)$,

$$
h(\omega)=\frac{V_{2}(\omega)}{V_{1}(\omega)}
$$

B. Measurement setup

The actual measurement of $h(\omega)$ is performed using a suitable vector network analyzer (VNA) in combination with two identical passive voltage probes having the same transfer function $K(\omega)$, see left part of Fig. 4. We use a VNA setting with $1 \mathrm{M} \Omega$ input impedance for the VNA reference (R) and input (T). The signal is brought from the VNA to the transformer terminal using a coaxial cable. The VNA itself is separated galvanically from the power outlet using a separation transformer, to minimize interference.

When the two probes are identical (and of equal length), the measured voltage ratio on the VNA ( $\left.V_{T} / V_{R}\right)$ is exactly equal to $V_{2} / V_{1}$, since we have

$$
h(\omega)=\frac{V_{T}(\omega)}{V_{R}(\omega)}=\frac{K(\omega) \cdot V_{2}(\omega)}{K(\omega) \cdot V_{1}(\omega)}=\frac{V_{2}(\omega)}{V_{1}(\omega)}
$$

The measured response is of course dependent on the terminal condition of the other terminals (open, grounded or loaded).

In order to connect to the transformer terminals by coaxial cables, prefabricated test fixtures (connections) were used, see right part of Fig. 4. They include an N-type coaxial connection to which a grounding shunt can be screwed for fast grounding or opening of terminals. The voltage probe (tip and grounding clips) are easily connected to the test fixture.

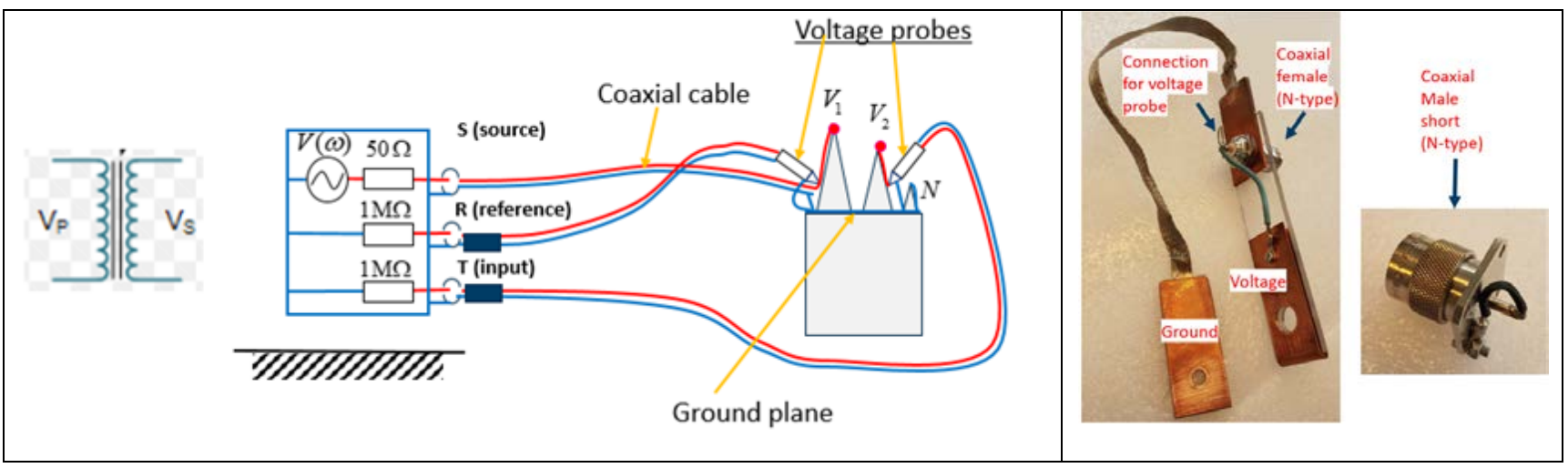

Figure 4: Left: Voltage transfer measurement. Red: voltage signal conductors; blue: ground conductors/grounded parts.

Right: Test fixture for attaching coaxial cable and grounding shunt.

C. Measurement Conditions

Similarly, as in [3], a ground plane is established on the transformer that is used as a ground reference. The ground reference is made from braided wire that is connected to grounding points on the transformer. Fig 5 shows the terminations used in the measurements as well as the ground reference. In this case, we used two external terminals (H3, X3) while all other external terminals were grounded (left part of Fig. 5). Additionally, we measured voltage transfer to three internal points in the regulating winding (leads marked 3, 5, 6 in Fig. 5). 


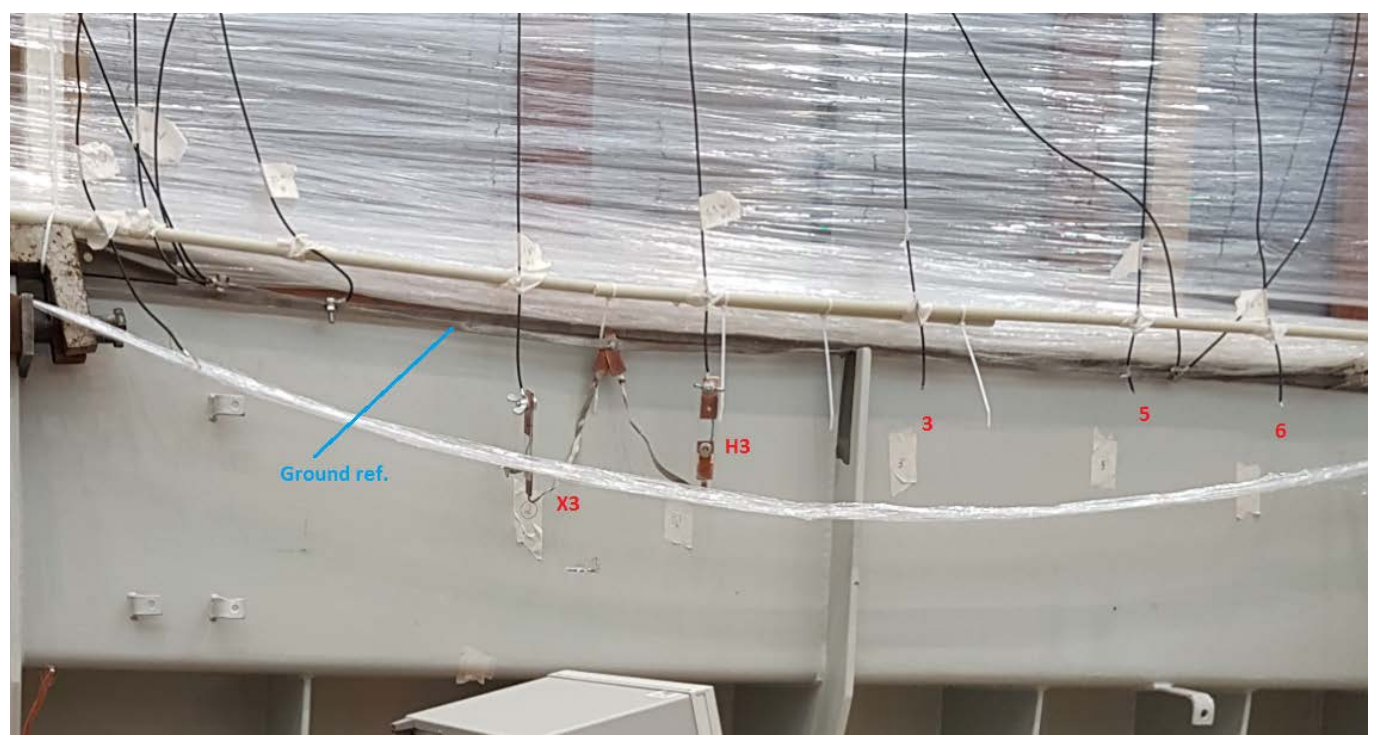

Figure 5: Connections. Voltage transfer measurements from floor level.

D. Conversion from frequency domain to time domain

Fig. 6 (left panel) shows an example of measured voltage transfer functions, from X3 to P3, P5 and P6, with H3 grounded. The left panel shows the magnitude functions (blue traces - "Data"), as well as a rational function approximation calculated by vector fitting (dashed red traces - "FRVF"). The rational function (3) is seen to match the measurement very closely.

$$
h(\omega) \cong r_{0}+\sum_{i=1}^{N} \frac{r_{i}}{j \omega-a_{i}}
$$

Using the rational approximation (3), which defines an impulse response, the time domain response of any time domain excitation can be calculated using "recursive convolution" via time domain discretization. Details of the rational fitting and time domain convolution is found in [3] and references therein. The right panel in Fig. 6 shows the 1.2/50 $\mu$ s lightning impulse response as calculated from the rational approximations (3) associated with the frequency responses in the left panel of Fig. 6.
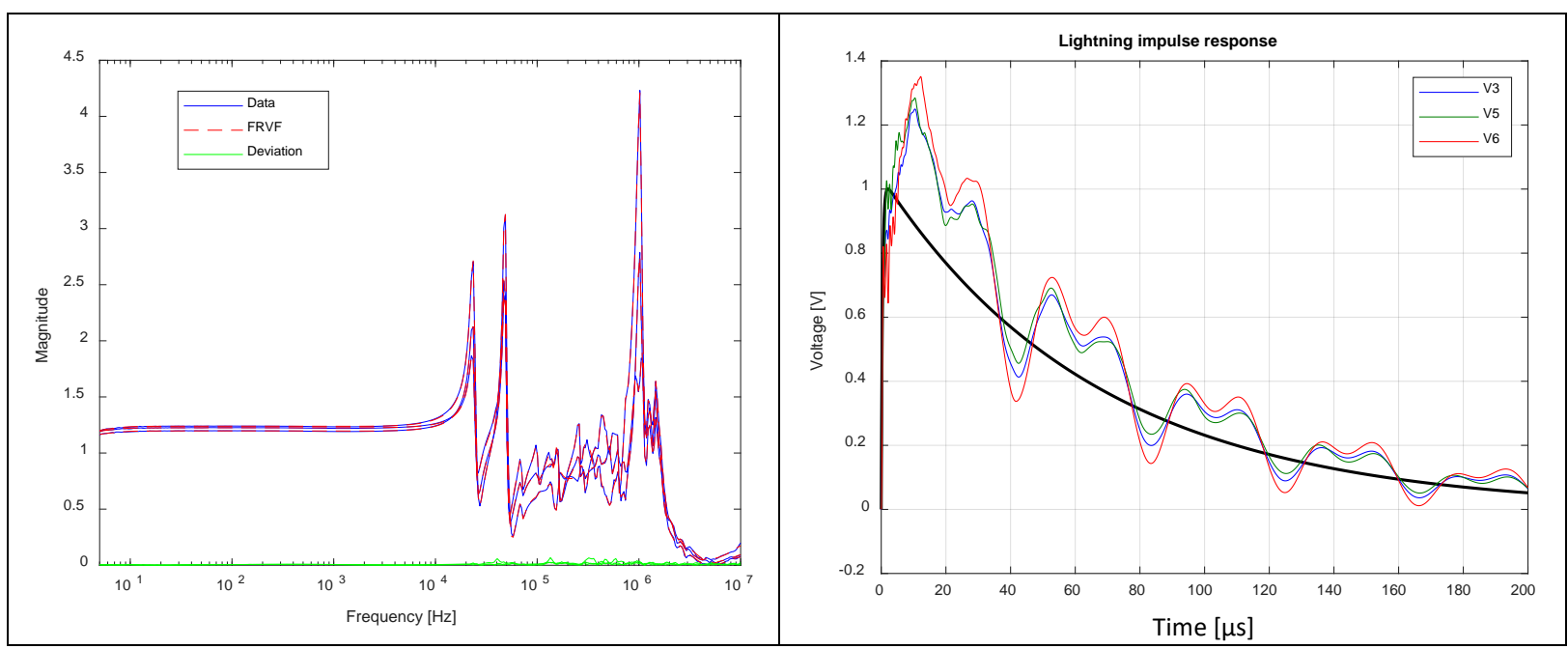

Figure 6: Left: measured voltage transfer functions and a $60^{\text {th }}$ order rational approximation; right: calculated 1.2/50 $\mu$ s voltage responses using recursive convolution. Case 3.

The accuracy of the procedure was verified by applying a time domain step voltage to one terminal and measuring the voltage responses using an oscilloscope. The recursive convolution approach was able to reproduce the measured voltage waveforms with excellent agreement. Fig. 7 shows one example of comparison. A zoomed view of the response is shown in the right panel. 


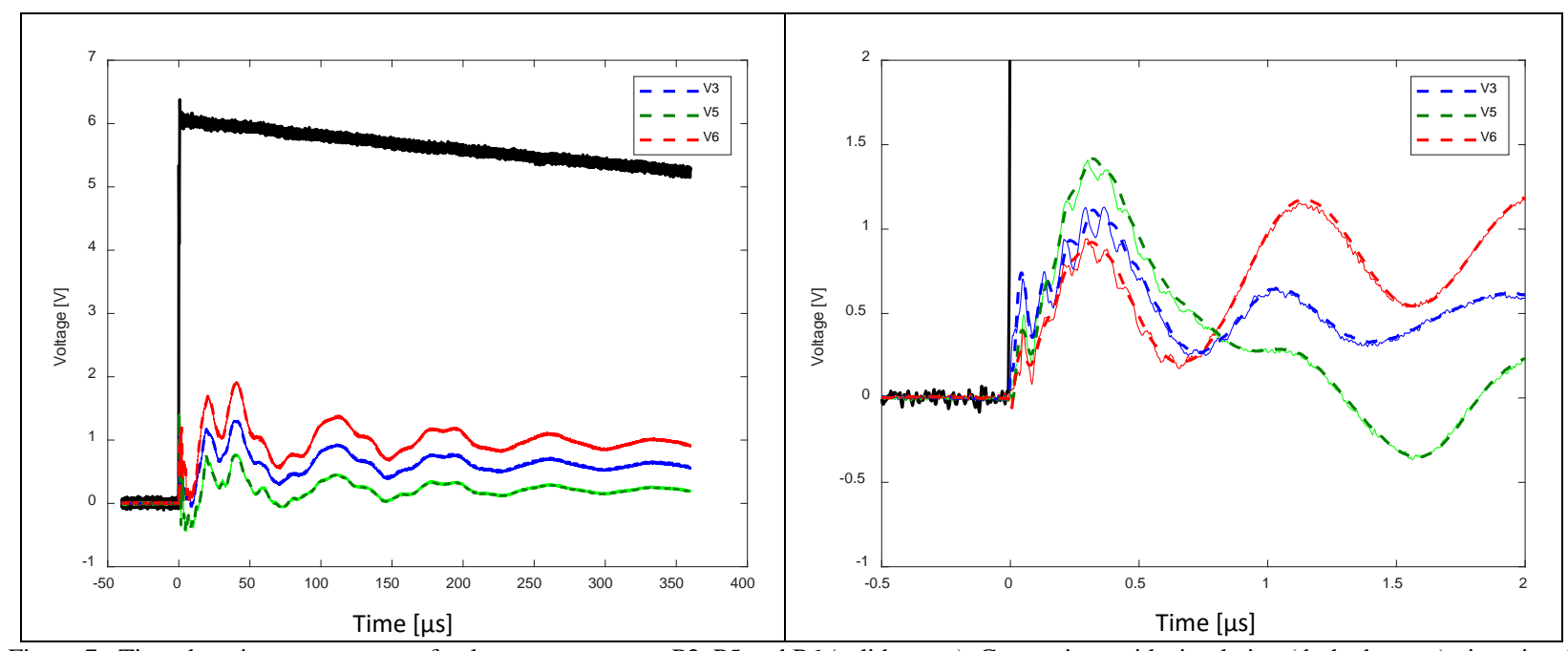

Figure 7: Time domain measurement of voltage responses on P3, P5 and P6 (solid traces). Comparison with simulation (dashed traces) via rational model and recursive convolution.

\section{WHITE-BOX CALCULATIONS VS MEASUREMENTS}

\section{A. Outline of the simulation procedure}

The applied white-box model is a lumped-parameter type equivalent circuit composed of resistances, capacitances, self-inductances and mutual inductances. The model is intended to emulate the transformer response to high frequency transients as close as possible to reality. We start by splitting the coils into the portions of interest, depending on the desired accuracy, not forgetting that the higher the number of lumped parameters the longer it will take to solve the equivalent circuit. The ends of these portions, which feature lumped parameters, will be the nodes of the equivalent circuit.

This discretization procedure implies the determination of the self and mutual inductance matrix, the resistive elements between each one of the nodes and the capacitances of the various coils' portions.

The capacitances calculations include the following elements:

- $\quad$ Between turns;

- Between coils;

- Between coils and static plates;

- Between static plates;

- Between coils and ground (magnetic circuit, magnetic shielding and tank walls);

- Between static plates and ground (magnetic circuit, magnetic shielding and tank walls);

The determination of all capacitances and resistances are made by analytical formulae. The self and mutual inductances are determined using a 3D numerical software. Finally, the RLC circuit is built and solved using a circuit analysis program.

This calculation tool is continuously being improved by Efacec and allows to control every circuit parameter and to refine it when necessary. It is also being automated and integrated in Efacec's information system to accelerate the transient simulations and avoid human errors.

\section{B. Leads' connections}

Several test cases were made with alternative leads (terminal) conditions. Table I and Fig. 8 define the leads connections, taking Fig. 1 as reference for the nodes' designation.

TABLE I: LEADS CONNECTIONS

\begin{tabular}{|c|c|c|c|c|c|}
\hline Name & $\begin{array}{c}\text { H3 Lead } \\
(1.1 \mathrm{~W})\end{array}$ & $\begin{array}{c}\text { X3 Lead } \\
(2.1 \mathrm{~W})\end{array}$ & $\begin{array}{c}3.2 \text { Lead } \\
(3.2 \mathrm{~W})\end{array}$ & $\begin{array}{c}3.1 \text { Lead } \\
(3.1 \mathrm{~W})\end{array}$ & $\begin{array}{c}\text { H0X0 Lead } \\
(2 \mathrm{~W})\end{array}$ \\
\hline Case 01 & Applied & Grounded & Grounded & Grounded & Grounded \\
\hline Case 02 & Applied & Open & Grounded & Grounded & Grounded \\
\hline Case 03 & Grounded & Applied & Grounded & Grounded & Grounded \\
\hline Case 04 & Open & Applied & Grounded & Grounded & Grounded \\
\hline Case 05 & Applied & Open & Open & Grounded & Grounded \\
\hline Case 06 & Applied & Grounded & Open & Grounded & Grounded \\
\hline
\end{tabular}




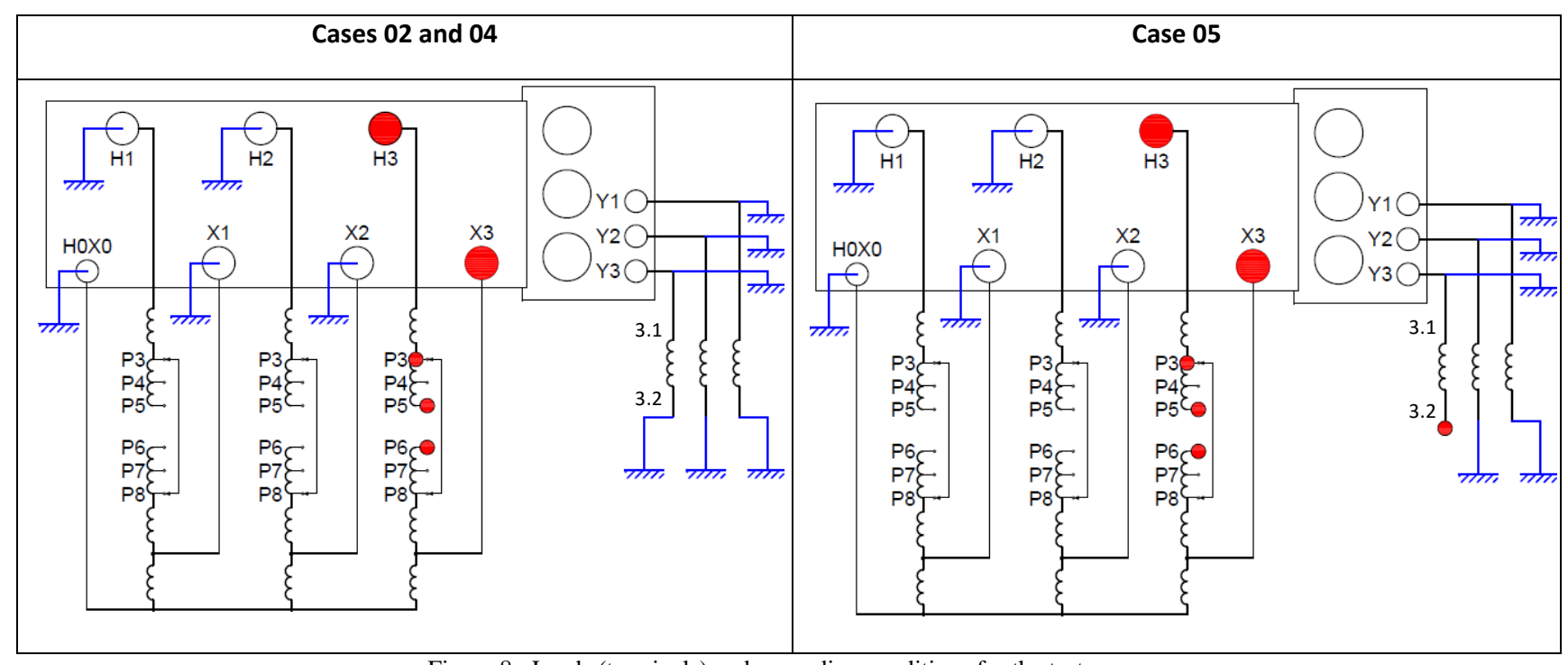

Figure 8: Leads (terminals) and grounding conditions for the test cases.

\section{Measurements vs simulations}

Fig. 9 to Fig. 18 show the comparison between the calculated values and the measured results. One can in all cases observe a very good agreement between calculated and measured values.

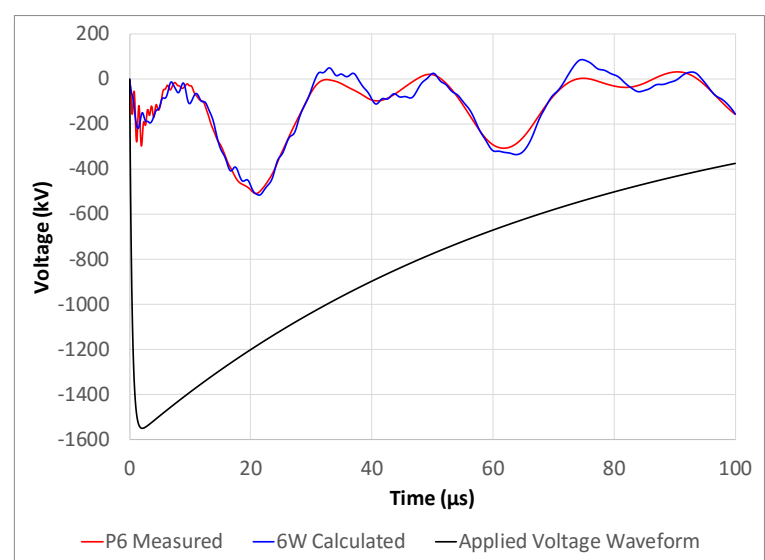

Figure 9: Comparison for Case 01, P6 lead, (black: applied waveform, red: measurement, blue: calculation)

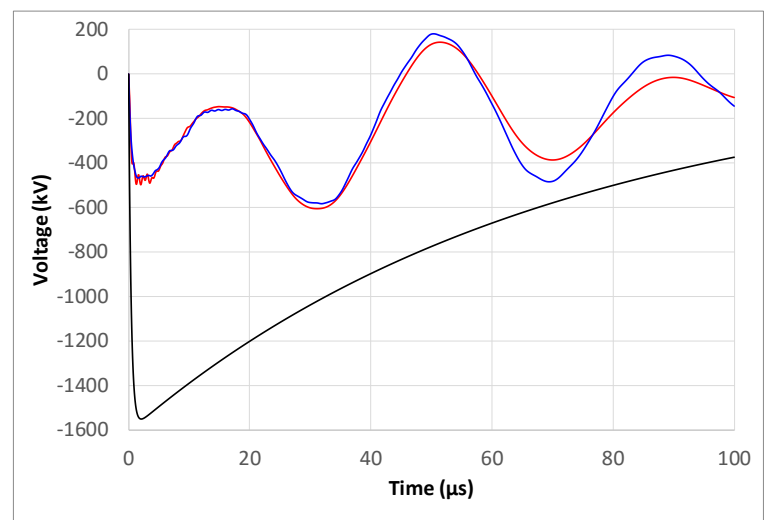

—X3 Measured - 2.1W Calculated -Applied Voltage Waveform

Figure 11: Comparison for Case 02, X3 lead, (black: applied waveform, red: measurement, blue: calculation)

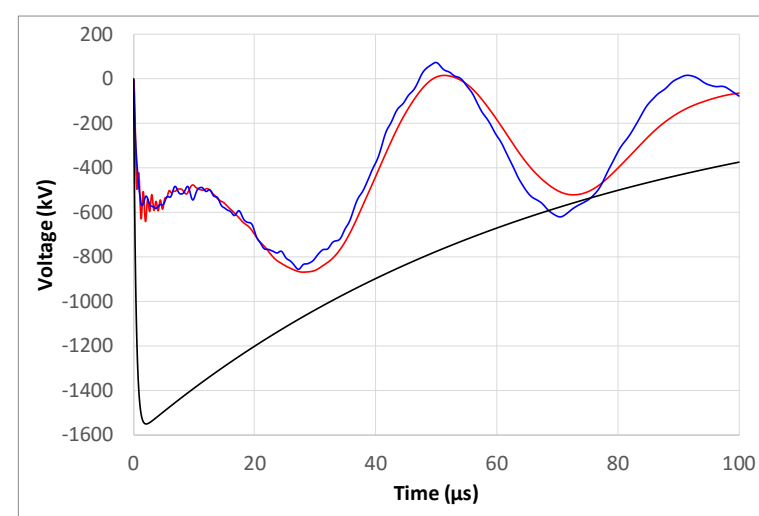

—P6 Measured —6W Calculated —Applied Voltage Waveform

Figure 10: Comparison for Case 02, P6 lead, (black: applied waveform, red: measurement, blue: calculation)

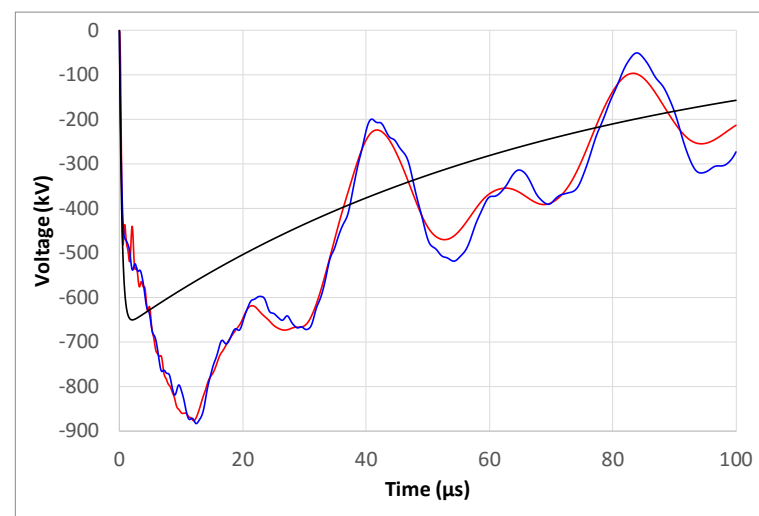

-P6 Measured —6W Calculated —Applied Voltage Waveform

Figure 12: Comparison for Case 03, P6 lead, (black: applied waveform, red: measurement, blue: calculation) 


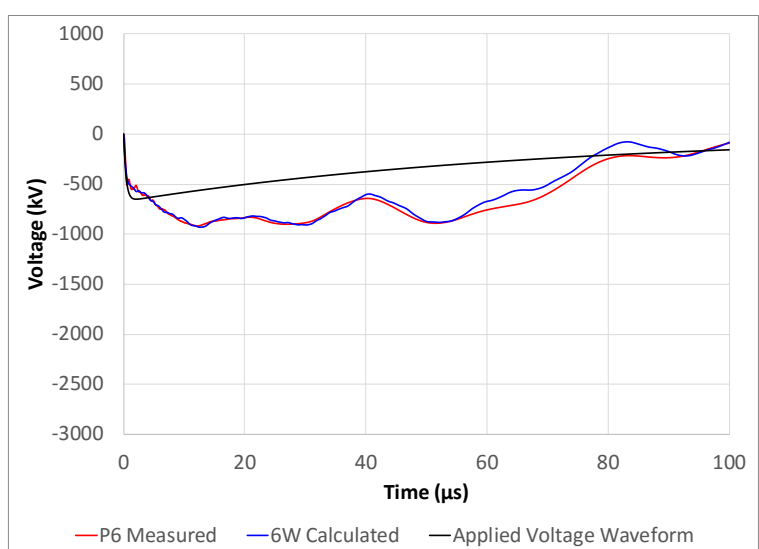

Figure 13: Comparison for Case 04, P6 lead, (black: applied waveform, red: measurement, blue: calculation)

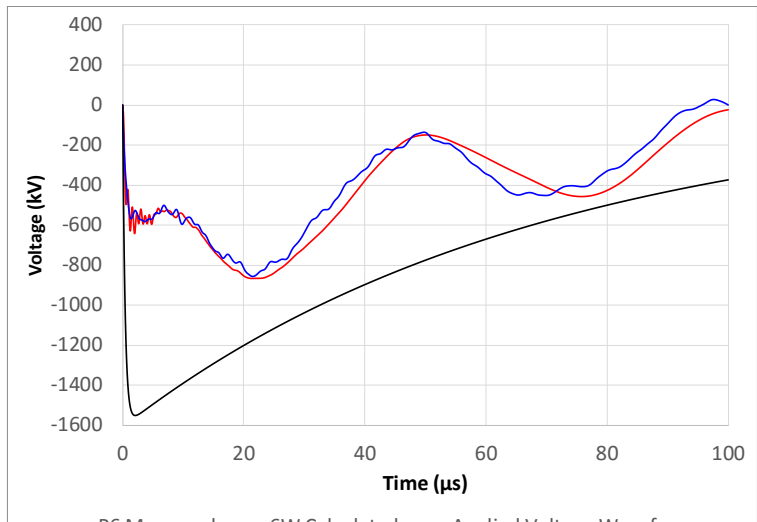

—P6 Measured —6W Calculated —Applied Voltage Waveform

Figure 15: Comparison for Case 05, P6 lead black: applied waveform, red: measurement, blue: calculation)

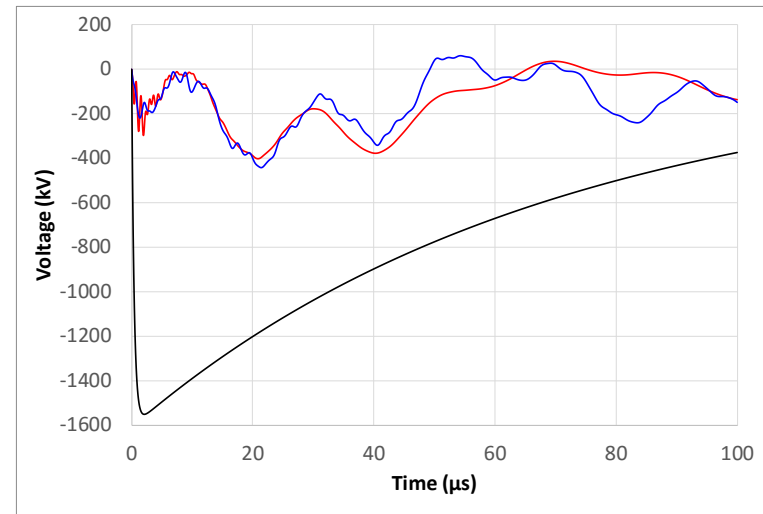

—P6 Measured —6W Calculated -Applied Voltage Waveform

Figure 17: Comparison for Case 06, P6 lead (black: applied waveform, red: measurement, blue: calculation)

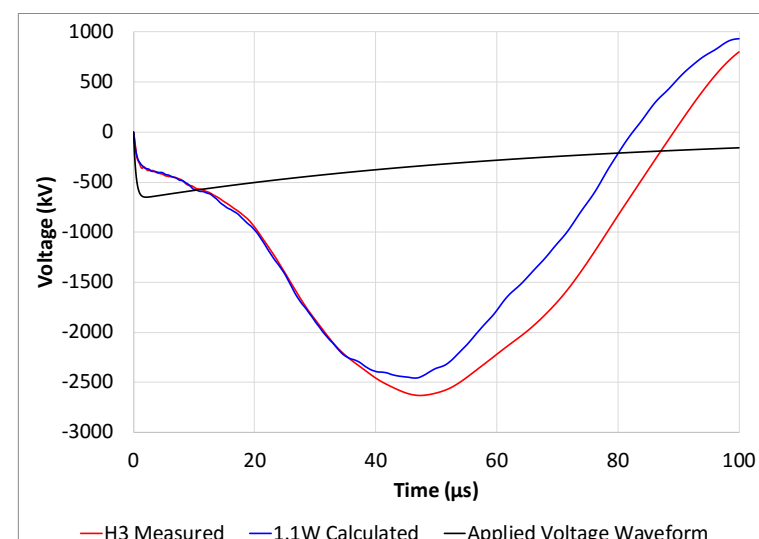

- H3 Measured -1.1W Calculated -Applied Voltage Waveform

Figure 14: Comparison for Case 04, H3 lead, (black: applied waveform, red: measurement, blue: calculation)

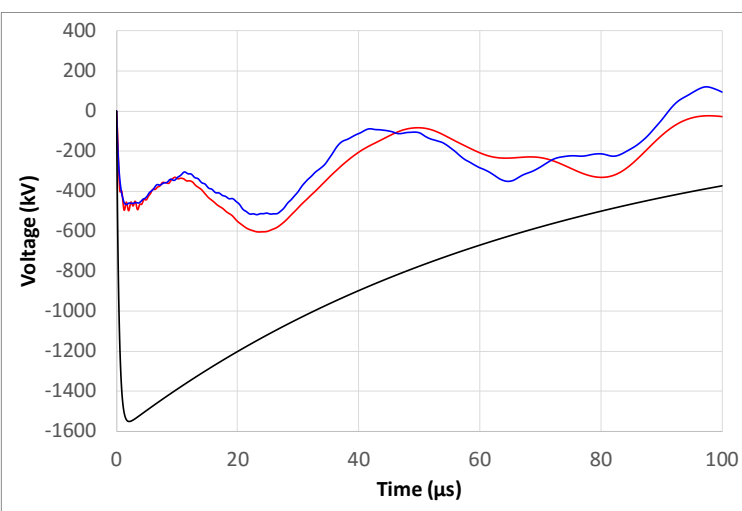

—X3 Measured -2.1W Calculated —Applied Voltage Waveform

Figure 16: Comparison for Case 05, X3 lead, (black: applied waveform, red: measurement, blue: calculation)

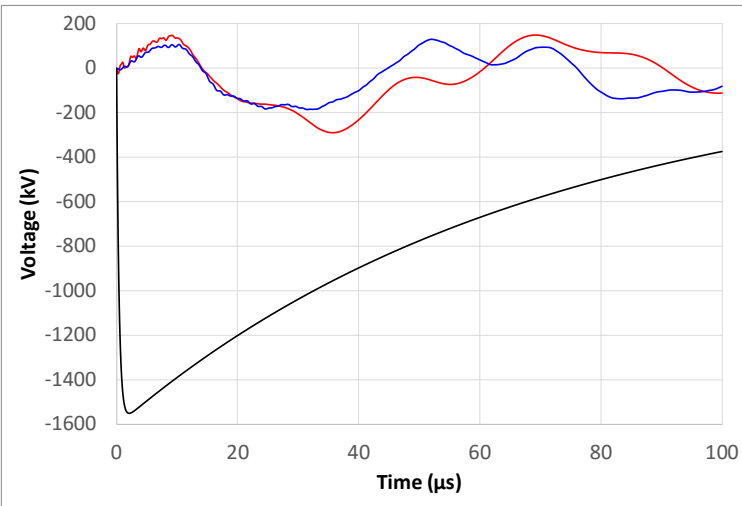

-3.2 Measured - 3.2W Calculated - Applied Voltage Waveform

Figure 18: Comparison for Case 06, 3.2 lead (black: applied waveform, red: measurement, blue: calculation)

\section{OBSERVATIONS ON MEASUREMENT ACCURACY}

The measurement points (H3, X3, P3, P5, P6) were brought down from the transformer's windings to the ground reference using insulated wires, as can be observed in Fig. 5. The presence of such wires can potentially lead to false oscillations which manifest as (false) resonances at high frequencies in the measured transfer functions. In order to clarify this potential problem, a set of duplicate measurements were performed where the measurements were made from a platform. This permitted the lengths of the insulated wires to be reduced by approximately $1.5 \mathrm{~m}$. The ground reference was elevated $1.5 \mathrm{~m}$ as well.

Fig. 19 shows the same result for time domain voltage transfer functions as in Fig. 6. The result with shorter leads is shown with dashed traces, superimposed on the original responses (solid traces). The traces are virtually overlapping, except for the initial response (right panel). It is observed that reducing the lead lengths by $1.5 \mathrm{~m}$ affects the high-frequency oscillation component of about $1 \mathrm{MHz}$. Reducing the length slightly increases the oscillation frequency while the amplitude is reduced. 


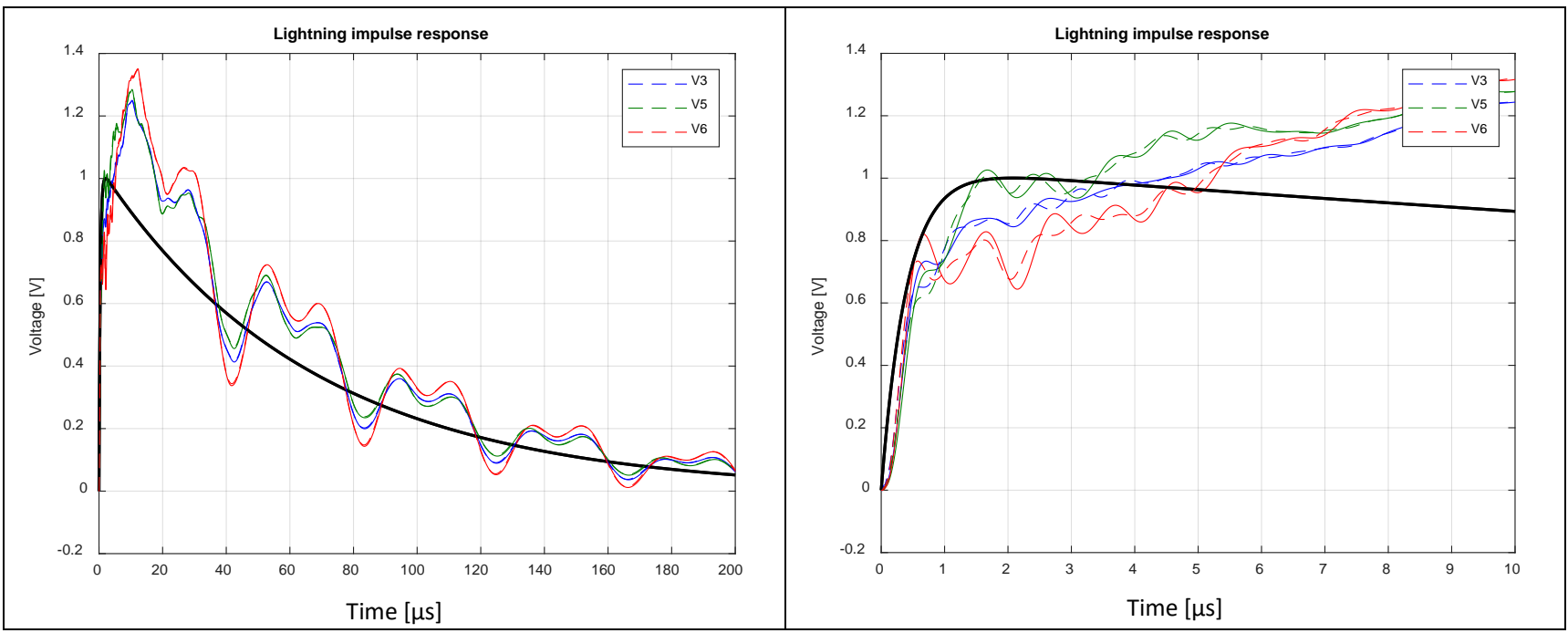

Figure 19: Effect of reducing the connection leads lengths by $1.5 \mathrm{~m}$. Results with shorter leads (dashed traces) and with longer leads (solid traces). Right panel: zoomed view of left panel.

\section{DISCUSSION}

The accuracy of white-box simulations (Figs. 9-18) are in general better than similar simulations obtained for a core-form transformer [3]. One reason can be that shell-form transformers are inherently easier to model than core-form transformers, due to lower complexity and higher series capacitance:

- Shell-form transformers have almost no magnetic or capacitive coupling between phases, unlike core-form transformers;

- Shell-form transformers have normally a reduced number of coils when compared to core-form transformers and have basically two types of windings (continuous coil winding and parallel wound tap coil winding) while in core-form transformers we can find several types of windings (continuous winding, a few types of interleaved discs winding, shielded-conductor winding, layer winding, etc.);

- Also, in shell-form transformers one normally finds a more favorable ratio between the series capacitance and the ground capacitance (higher $k$ ) than in core-form transformers, which improves the voltage distribution and its predictability. In fact, looking at Fig. 3, one observes that the voltage distribution has higher sensitivity to lower $k$ values than to higher $k$ values. For instance, changing $k$ from 10 to 1 worsens much more the voltage distribution than changing $k$ from 100 to 10 .

\section{CONCLUSION}

A non-standard impulse test procedure was used for obtaining the voltage response at critical positions in the regulating winding, with alternative terminal conditions. The measured responses were compared to simulations by a white-box model, demonstrating a good accuracy between measurements and simulations. The measurements are in general fast and easy to perform. The approach is an excellent means of assessing the accuracy of a manufacturer's impulse voltage computational tools, thereby enabling further improvements to the model's parameter determination.

\section{ACKNOWLEDGMENTS}

This article is a result of the project GreenEst - Green Ester Transformers, supported by Competitiveness and Internationalisation Operational Programme (POCI), under the PORTUGAL 2020 Partnership Agreement, through the European Regional Development Fund (ERDF). Additional support from Norwegian Research Council, (project "ProTrafo", no. 207160/E20) is appreciated.

\section{REFERENCES}

[1] IEC 60076-3, Power transformers - Part 3: Insulation levels, dielectric tests and external clearances in air.

[2] CIGRE Technical Brochure 577A, "Electrical transient interaction between transformers and the power system. Part 1 - Expertise", CIGRE JWG A2/C4.39, April 2014.

[3] B. Gustavsen, A. Portillo, R. Ronchi, A. Mjelve, "Measurements for validation of manufacturer's white-box transformer models", 4th International Colloquium Transformer Research and Asset Management, May 10-12, 2017, Pula, Croatia. Procedia Engineering 202 (2017), pp. 240-250. 